\title{
Erratum to: A Simple Explicit Formula for the Voigt-Reuss-Hill Average of Elastic Polycrystals with Arbitrary Crystal and Texture Symmetries
}

\author{
Chi-Sing Man • Mojia Huang
}

Published online: 21 October 2011

(C) Springer Science+Business Media B.V. 2011

\section{Erratum to: J. Elast (2011) 105:29-48}

DOI 10.1007/s10659-011-9312-y

Equation (67) on page 44 should read:

$$
\begin{aligned}
\boldsymbol{C}^{H}(w)= & \frac{1}{2}\left(\boldsymbol{C}^{V}(w)+\boldsymbol{C}^{R}(w)\right) \\
= & \frac{1}{2}\left(\boldsymbol{C}_{\mathrm{iso}}^{V}+\boldsymbol{C}_{\mathrm{iso}}^{R}\right)+\sum_{k=2,4} \sum_{m, n=-k}^{k} \frac{2 \pi^{2}}{(2 k+1)}\left(4 \gamma_{n}^{k, 1}-\frac{1}{\mu_{s}^{2}} \zeta_{n}^{k, 1}\right) c_{m n}^{k} \boldsymbol{H}_{m}^{k, 1} \\
& +\sum_{m, n=-2}^{2} \frac{4 \pi^{2}}{5}\left(2 \gamma_{n}^{2,2}-\frac{1}{\mu_{s}\left(3 \lambda_{s}+2 \mu_{s}\right)} \zeta_{n}^{2,2}\right) c_{m n}^{2} \boldsymbol{H}_{m}^{2,2} .
\end{aligned}
$$

The parameters $\gamma_{n}^{k, 1}$ and $\zeta_{n}^{k, 1}$ in the preceding formula were erroneously put in the original article as $\gamma_{n}^{k, s}$ and $\zeta_{n}^{k, s}$, respectively. The assertion there that these parameters are given, respectively, by (54) and by the counterpart of (54) with $\hat{c}_{\alpha \beta}$ replaced by $\hat{s}_{\alpha \beta}$ remains valid.

The online version of the original article can be found under doi:10.1007/s10659-011-9312-y.

C.-S. Man ( $\otimes)$

Department of Mathematics, University of Kentucky, Lexington, KY 40506-0027, USA

e-mail: chi-sing.man@uky.edu

M. Huang

Institute for Advanced Study, and Institute of Engineering Mechanics, Nanchang University, Nanchang, Jiangxi 330031, China

e-mail: mojiahuang@ hotmail.com 